\title{
Exploring the Space-Calorie Association: Preliminary Evidence from Reaction Time Performance
}

\author{
Emre Gurbuz' and Ahu Gokce \\ ' Department of Psychology, Saarland University \\ 2 Department of Psychology, Kadir Has University
}

\section{KEYWORDS}

space-calorie association,

high and low-calories,

endogenous spatial cues,

approach-avoidance

mechanisms
ABSTRACT

The present study was designed to investigate the representation of calorie levels in space. Previously an association between numerical magnitude and space has been established, namely, the Spatial Numerical Association of Response Codes (SNARC) effect. The spatial-numerical association reveals representation of smaller and larger numbers by the left and right sides, respectively. In addition, previous studies showed that spatial arrangement of foods in space affects the food selection behavior. In three experiments, the presence of an association between calorie magnitude and space was tested to understand how it could potentially affect food selection behavior. Reaction times were recorded to investigate the speed of information processing. In Experiment 1, locations of low and high calorie food stimuli were (in)congruent in terms of the space-calorie association. In Experiment 2, endogenous spatial cues were used to bias attention to investigate if this bias would lead to formation of the space-calorie association. Finally, Experiment 3 investigated whether green and red colors evoke approach or avoidance behavior and prevent formation of the space-calorie association. In all experiments, results revealed lack of an association between space and calorie, that is, presenting low/high calorie items on the left/right hemispace, respectively, did not modulate the processing speed.

\section{INTRODUCTION}

Spatial cognition studies mainly focus on how the items (e.g., objects in the environment, stimuli in the visual display, food images on the menus) are represented in space. By employing various paradigms and tasks, different aspects of spatial representations can be uncovered. The spatial numerical association of response codes (SNARC) effect reflects the association between the number magnitude and space (Dehaene et al., 1993). Numbers are represented in ascending order from left to right on the horizontal mental number line, resulting in associating the numbers in smaller magnitude with the left side and numbers in larger magnitude with the right side on the horizontal layout. Moyer and Landauer (1967) investigated the horizontal numerical representation in a comparison judgment task. Participants were presented with single-digit number pairs where each digit was presented on the left and right sides and were asked to indicate the location of the larger digit in the pair. Responses were faster and error rates were smaller for pairs with the larger numerical difference (e.g., 3 and 9) compared to pairs with less numerical difference (e.g., 7 and 9). The comparison judgment task was easier to perform when the numbers were more distant on the mental number line compared to closer distance (Moyer \& Landauer, 1967). Differences in performance as a function of the numerical magnitude difference reported by Moyer and Landauer (1967) support the idea of the spatial representation of numbers.

Corresponding author: Ahu Gokce, Department of Psychology, Kadir Has University, Cibali Mah. Hisaralti Cad. No: 17, 34083 Istanbul, Turkey.

E-mail:ahu.gokce@khas.edu.tr 
The performance superiority for the larger magnitude differences in number pairs indicates the left-right hemispace representations of the smaller and larger numbers, respectively. The formation of an association between the location of the response hand (left, right) and the magnitude of the number has been proposed as the underlying mechanism of the SNARC effect. The right-hand responses were faster for the larger numbers while left-hand responses were faster for the smaller numbers (Dehaene et al., 1993). Such an association leads to representation of the smaller numbers on the mental number line with left hand responses and larger numbers with the right-hand responses. Interestingly, this tendency was also observed when the evaluation of the numerical magnitude was task-irrelevant in a parity judgment task and was not affected by the handedness of the participants (Dehaene et al., 1993; Experiment 5).

An alternative explanation for the SNARC effect was provided by the polarity correspondence view (Proctor \& Cho, 2006), according to which dimensions include "-" and “+” polarities and stimuli or responses can be assigned to either polarity. In the domain of the numerical magnitude, right-hand responses and larger numbers indicate the "+" side and left-hand responses and smaller numbers indicate the "_" side of the polarity. The match between the polarities of the stimulus and response (e.g., both “-” polarities) leads to faster processing speed. Non-numerical magnitudes such as size, luminance, time, and tone can be polarized as well. Bigger sizes, darker colors, future times, or higher pitches represent the "+" side of the polarity whereas smaller sizes, lighter colors, past times, and lower pitches represent the "-" side of the polarity. However, in a meta-analysis, it was discussed that these explanations are not mutually exclusive and that both the mental number line and the polarity correspondence views can account for the SNARC effect (Wood et al., 2008).

Space-magnitude associations have been reported in various nonnumerical domains such as time, size, letters, and luminance, known as the SNARC-like effect (Fumarola et al., 2014; Sellaro et al., 2015). In the temporal domain, earlier and later magnitudes were associated with the left and right side of the hemispace, respectively (Macnamara et al., 2018). Similarly, individuals responded faster to the earlier months of the year with the left hand and the latter months of the year with the right hand (Gevers et al., 2003; see also Torralbo et al., 2006). In the musical domain, lower/higher pitch sounds were associated with the left/right hand, respectively (Cho et al., 2012; Nishimura \& Yokosawa, 2009). Ren et al. (2011) asked participants to compare two stimuli based on their size (bigger or smaller), luminance (darker or lighter), and conceptual size (bigger or smaller, e.g., mountain or dust) in various experiments. Right hand responses were faster for larger, darker, and conceptually bigger targets compared to smaller, lighter, and conceptually smaller targets (see also Dural et al., 2018).

The aim of the current study was to test whether an association between space and calorie magnitude (i.e., level) exists. Various features of food calories can be investigated such as healthiness (Manippa et al. , 2020; Romero \& Biswas, 2016) or (visual) heaviness (Deng \& Kahn, 2009) of certain foods regardless of the calorie level. The present study's focus was on the representation of the calorie level (in kcal) of the presented food item in the left/right space. Investigation of this issue is important to be able to understand and explain certain factors as to why people choose to consume certain foods. In this regard, previous studies reported that the spatial arrangement of foods in space has a role in the selection of the food. For instance, when the food item was in far proximity and harder to reach, people chose that option less compared to when located closer in a salad bar (Rozin et al., 2011). Similarly, Romero and Biswas (2016) showed that lateral positioning of healthy/unhealthy foods influenced the food selection behavior. In Experiment 1, participants were provided with two kinds of menus in which the healthy item was presented on the left and the unhealthy item on the right and the reversed order was presented in the second menu. Healthy food preference increased when the healthy item was located on the left side of the unhealthy option (Romero \& Biswas, 2016). Results were replicated in follow-up experiments using a computer-based task (classifying and placing low and high calorie items in left/right sided boxes) and in real-life context where participants' consumption of healthy beverages was investigated. The authors concluded that positioning the healthy food congruent with the natural representation of the left and right space increases the amount of the healthy food choices. Deng and Kahn (2009) showed that similar bias exists on the vertical layout. The authors investigated the effect of product image location on visual heaviness perception of the product package. When the product images were located at the bottom, products were perceived as heavier compared to when located at the upper part of the package. Interestingly, this effect was not observed for the non-food items. This has been explained by the view that heaviness represents more quantity for foods but means danger for the non-food items. Additionally, the authors put forward the idea that the pull of the heavy items down by the gravitational forces creates bottom-heavy bias (see also Tversky, 2011). Such a bias can lead to the formation of an association between heavy items and bottom locations. Furthermore, it has been suggested that certain beliefs of individuals implicitly play a role in their consumption behavior. In series of experiments, Valenzuela and Raghubir (2015) investigated position-based effects in the horizontal and vertical arrangement of products on the shelves. Results indicated that consumers have certain beliefs that the products are placed based on their price on both the vertical (cheaper products at the bottom shelves) and horizontal (cheaper products on the left shelves) axes.

The effect of laterality bias on the (disentangled) representations of the healthiness and caloric content features was further examined in a recent study by Manippa et al. (2020). In Experiment 1, healthiness (unhealthy or healthy) and caloric content (low or high) dimensions were manipulated and participants' preference (frequency in percentages) to categorize food items on these dimensions was measured. In the task, the four labels that derived from the two dimensions were randomly located on each corner and the to-be-categorized food item was located in the center of the screen. The highest frequency of the preference was observed for the healthy label followed by the high-calorie label indicating that people prefer to categorize foods mostly based on their healthiness. Furthermore, laterality bias was 
observed for the caloric content dimension: High-calorie preference was the highest when the label was on the right side (upper/lower right corners), which is in accordance with the mental number line representation. In Experiment 2, the visual analog scale (VAS) was used to assess participants' ratings of food items on healthiness and caloric content dimensions. The labelling positions (left: healthy/high-calorie and right: unhealthy/low-calorie) were randomized across trials leading to (in)congruent arrangements of space and healthiness/calorie content in terms of the mental number line. The healthiness ratings were higher in the congruent arrangement condition, that is, when the "healthy" label was on the left side of the VAS. Such pattern was not observed for the calorie content. The authors also examined reaction time performance (Experiment 3 ) in a pleasantness evaluation task. The task was based on evaluating the (un)pleasantness of each presented target food item. Pleasantness was operationalized as being edible (i.e., pleasant) or rotten (i.e., unpleasant). Response mappings (left/right response key) for the pleasantness ratings were reversed for two groups of participants. Results revealed an opposite pattern of the SNARC-like effect, pleasantness ratings were faster for the right-side responses compared to the left side.

The results reported by Manippa et al. (2020) overall indicate the superiority of the healthiness dimension over the caloric content in a food categorization task. Although the healthiness aspect plays a critical role when individuals classify food, it remains unclear whether calorie level estimation is done accurately. Judging the food as healthy could lead to an underestimation of its calorie level (Chandon \& Wansink, 2007). In order to test this issue more precisely, the present study was designed to investigate the space-calorie association by controlling the calorie level. Processing speed (i.e., RT) was measured, which provided information on the mental chronometry (Meyer et al., 1988) and enabled to precisely measure information processing performance in a computerized setting. Based on the polarization view (Proctor \& Cho, 2006), different calorie amounts can be represented at the opposite sides of the polarity. While higher calorie foods were expected to be on the "+" side of polarization and associated with the right side, low calorie foods were expected to be on the "-" side of the polarization and associated with the left side of the screen. Similarly, an increase in the calorie level can be represented on a horizontal mental number line where lower calorie levels are represented on the left and higher calorie levels are represented on the right side of the mental number line. The presence of the space-calorie association would result in representing low-calorie foods on the left and high-calorie foods on the right side. The space-calorie representation would lead to improved task performance, measured by the processing speed, when the lowand high-calorie food stimuli are presented on the left and right sides, respectively. Reaction time measurement would reflect the association between the calorie level and space in terms of faster reaction times when the low/high calorie item is placed congruently. The investigation of the space-calorie association is important based on several factors. Firstly, testing if low and high calorie levels are represented in the left/ right hemispaces, respectively, would enable us to extend our knowledge on the SNARC-like effect within the calorie domain. Secondly, the demonstration of such an association would have practical implications in applied settings, for instance, when developing intervention programs in the scope of eating behaviors. This association was tested in three experiments by using different parameters. Experiment 1 was designed to test if the space-calorie association exists and affects the representation of different calorie levels (low, high) in space. In the following experiments, the effects of spatial cue (Experiment 2) and approach-avoidance mechanisms (Experiment 3) were tested. In all experiments, reaction time (RT) and error rates were the dependent variables.

\section{GENERAL METHOD}

The general setup of Experiments 1-3 was identical, with the exceptions noted in the Procedure section of each experiment.

\section{Participants}

Fifty-four participants in total (female: 42 , right-handed: $43, M_{\text {age }}=$ 20.21, $S D_{\text {age }}=2.51$; Experiment 1: 17, Experiment 2: 18, Experiment 3: 19 participants) participated in the experiments. The study was approved by the Ethics Committee of the Kadir Has University. Prior to participation, participants gave their informed consent. Participants who were enrolled in the psychology undergraduate program received course credits for their participation. All participants had normal or corrected-to-normal vision. They were naïve to the purpose of the study and were debriefed after the participation.

\section{Apparatus \& Stimuli}

All experiments were built by using the PsychoPy Builder software (version 1.90.3; Peirce, 2007, 2009). Responses were recorded via keyboard placed in front of participants. The distance between the computer screen and the participants was approximately $60 \mathrm{~cm}$ and the screen resolution was set to $1920 \times 1080$ pixels. The experiment room was dimly lit.

The food stimuli were selected from the Food-pics database (Blechert et al., 2019; Blechert et al., 2014). In total, 286 food stimuli with low and high calorie values were chosen from the database. Prior to running the study, a pilot study was conducted to determine whether the food stimuli have comparable calorie levels (in kcal) for the sample of the present study using Qualtrics (Provo, UT, USA). The aim was to avoid culture-specific factors that may influence calorie judgments of the stimuli and that all food stimuli are recognizable by the sample. In the pilot study, the food stimuli were rated based on their calorie levels (low, average, or high). All stimuli were presented on the center of screen and participants were asked to decide which calorie group best represents the current food image. The calorie levels were " $0-100$ kcal," "100-250 kcal," and "250-500 kcal," representing low, average, and high calorie levels, respectively. Each item remained on the screen until the response was made. Data were collected from 53 individuals that matched our sample in terms of age. Based on the rating responses, food stimuli which had inconsistent ratings with the actual calorie levels were excluded (8.39\%). For example, nuts and cheese were rated 


\begin{tabular}{|c|c|c|c|c|c|c|}
\hline \multicolumn{7}{|c|}{$\begin{array}{l}\text { TABLE } 1 . \\
\text { Means and SDs of the } V \\
\text { ing Ratings of the Low } \\
\text { Food-pics Database }\end{array}$} \\
\hline & & \multicolumn{2}{|c|}{ Low calorie } & \multicolumn{3}{|c|}{ High calorie } \\
\hline & & $M$ & $S D$ & $M$ & $S D$ & $p$ \\
\hline \multirow{4}{*}{ Experiments 1 and 2} & Valence & 59 & 8.46 & 53.7 & 5.99 & $<.001$ \\
\hline & Arousal & 34.2 & 8.17 & 37 & 5.84 & .016 \\
\hline & Palatability & 60.2 & 11.2 & 60.2 & 7.64 & .989 \\
\hline & Craving & 37.7 & 12.6 & 38.6 & 7.53 & .585 \\
\hline \multirow{4}{*}{ Experiment 3} & Valence & 57.2 & 6.86 & 53.8 & 6.69 & .159 \\
\hline & Arousal & 33 & 8.76 & 35.3 & 4.89 & .368 \\
\hline & Palatability & 56.9 & 10.2 & 61.8 & 8.46 & .151 \\
\hline & Craving & 33.5 & 12.5 & 38.8 & 7.74 & .163 \\
\hline
\end{tabular}

Note. The $p$ values represent the differences between the low and high calorie stimuli on these parameters.

as low-calorie items although these foods are labeled as high-calorie in the Food-pics database. In addition, all rated pictures were separated into three different calorie groups based on being rated mostly as 0-100 $\mathrm{kcal}, 100-250 \mathrm{kcal}$, or $250-500 \mathrm{kcal}$. In order to use food stimuli that represented the low or high calorie levels for our sample, stimuli that were rated as average (100-250 kcal) were discarded (24.81\%). Finally, for every remaining stimulus (197), the percentage of agreement for each category was calculated. If half of the participants did not rate one stimulus as belonging to one category (e.g., less than $50 \%$ of raters rated the stimulus as low/high calorie), it was discarded from the stimulus set (total exclusion rate: 18.78\%). These exclusion criteria led to 80 low-calorie (e.g., apple, kiwi) and 80 high-calorie (e.g., muffin, chips) food stimuli in total (see Appendix A for the IDs of the selected stimuli). The selected pictures were compared on the palatability, craving, valence, and arousal factors provided in the Food-pics database (Blechert et al., 2019, Blechert et al., 2014, see Table 1).

In order to determine if the ratings of the stimuli were consistent across participants, inter-rater reliability analysis was performed by calculating the Fleiss Kappa (Fleiss, 1971). Kappa value for the 160 chosen pictures was 0.59 , which indicated moderate agreement (while the value of 0 indicates chance agreement, (Fleiss, 1971), 0.41-0.60 range indicates moderate agreement (Landis \& Koch, 1977).

\section{EXPERIMENT 1}

Experiment 1 investigated whether the low-calorie food stimuli (e.g., salad) and high-calorie food stimuli (e.g., hamburger) have a natural tendency to be represented in the left and right hemispace, respectively. The calorie-space association determined the (in)congruency of each experimental condition. Positioning the low-calorie stimuli on the left and high-calorie stimuli on the right side was congruent and the reversed pairing was hypothesized to lead to incongruency with regard to the calorie-space association. Therefore, the aim of this experiment was to examine whether presenting the low (high) calorie food stimulus on the left (right) side would improve the task performance in terms of increased processing speed and accuracy rates.

\section{Procedure}

A practice session (1 block, 20 trials) was run prior to the main experiment ( 4 blocks, 480 trials each). The data of the practice session was not recorded. In the practice session, instead of low- and high-calorie stimuli, pairs of pebbles and flowers were used to get acquainted with the task procedure using non-food stimuli. Participants were instructed to indicate the location of the flower pictures after the presentation of a red fixation cross or indicate the location of the pebbles after the presentation of a green fixation cross. The background color was set to black. In the experiment, each trial started with the presentation of a green or red fixation cross in the center for $700 \mathrm{~ms}$. Depending on the fixation cross color, participants were asked to localize the low-calorie (green) or high calorie (red) stimulus. The color of the fixation cross was randomized across trials with equal probability of occurrence. After the fixation cross, food pairs were presented for $1500 \mathrm{~ms}$ and the response period was $2000 \mathrm{~ms}$ (see Figure 1). Each food stimulus (size: $12^{\circ} \times 9^{\circ}$ ) was positioned $10^{\circ}$ away from the fixation cross location.

Participants performed a go/no- go task which enableds to measure response inhibition and reflects processing speed. In this task, the no-go trials play a critical role to prevent automatic responding which could lead to responding without judging the calorie level of the presented food. However, as the nature of the go/no go task requires to withhold responses in the no-go trials, participants would need to effortfully decide whether the presented food pairs have the same/different calorie levels which enables to investigate the space-calorie association (van Dijck \& Fias, 2011).

The food pairs were created by having four different combinations of low/high calorie food stimuli located on the left/right sides, presenting low calorie foods on the left and high calorie foods on the right (LH; e.g., salad-hamburger), high calorie foods on the left and low-calorie foods on the right (HL; e.g., hamburger-salad), high calorie foods on

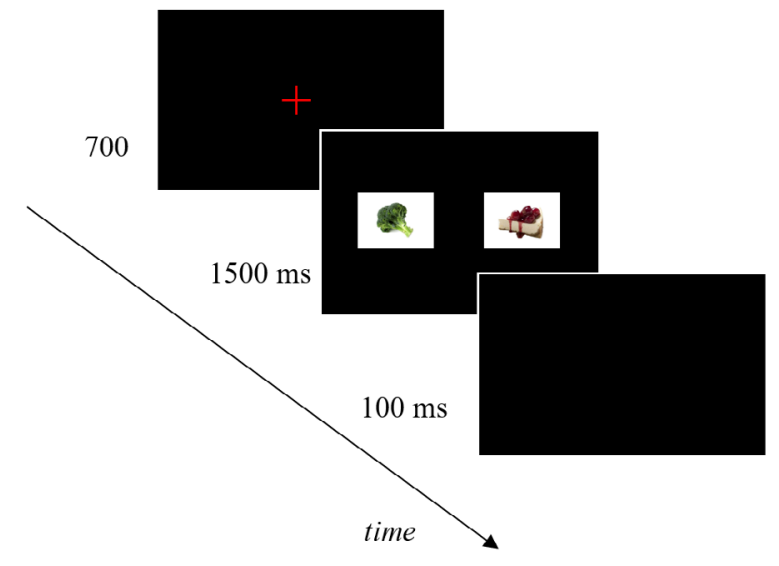

FIGURE 1.

The trial sequence of Experiment 1. Each trial started with the presentation of green or red fixation cross for $700 \mathrm{~ms}$, followed by the food stimuli for $1500 \mathrm{~ms}$. The task was to indicate the location of the low (high) calorie food stimulus after the presentation of the green (red) fixation cross. Here: the stimulus pair low-high (LH) was preceded by the red fixation cross, therefore participants had to indicate the location of the high calorie food (cheesecake). 
both sides (HH; e.g., hamburger-hamburger), or low-calorie foods on both sides (LL; e.g., salad-salad). The proportion of each pair was as follows: $40 \% \mathrm{LH}, 40 \% \mathrm{HL}, 10 \% \mathrm{LL}$, and $10 \% \mathrm{HH}$ pairs. In the $\mathrm{LH}$ and HL conditions ( $80 \%$ of the trials), participants were asked to respond to either low or high calorie item target (i.e., go trials). In the remaining $\mathrm{HH}$ and LL (20\% of the trials) conditions, no response was required as both items had the same calorie level (low-low or high-high) and there was no target to discriminate (i.e., no-go trials). In the go trials (i.e., $\mathrm{LH}$ and HL pairs), participants were asked to press the "A" or "L" button if the target was located on the left or right side, respectively. In the no-go trials (i.e., $\mathrm{LL}$ and $\mathrm{HH}$ pairs), participants were instructed to withhold their response. The HL and LH food stimuli pairs were pseudorandomized. The experiment session lasted approximately 30 minutes.

\section{Results and Discussion}

Data were analyzed by using R (R Development Core Team, 2017) and Jamovi (The Jamovi Project, 2019). In addition, JASP software (JASP Team, 2019) was used for the Bayesian statistical analyses. Bayes factor analyses were carried out as both the presence and the absence of the effects were of theoretical interest. The JASP software default parameters were used, with BF10 based on a directional H1 (pre > post) and a Cauchy prior width of 0.707 (Marsman \& Wagenmakers, 2017). A Bayes factor of $>3$ is considered as substantial evidence against the absence of effects, while a Bayes factor of $<1$ is evidence for the null effect.

Data of three participants were excluded from the analyses due to failure to follow instructions. Incorrect responses (20.85\%) and trials with $\pm 2.5 \mathrm{SDs}$ from the RT mean were discarded as outliers (1.3\%). The no-go trials ( $\mathrm{HH}$ and LL pairs) were not included in the analyses. In order to test the effect of pair congruency ( $\mathrm{LH}, \mathrm{HL}$ ) on target localization, paired $t$ tests were conducted for RTs and error rate variables separately. Reaction time, $t(13)=.22, p=.82$, Cohen's $d=.06$, and error rates, $t(13)$ $=0.78, p=0.44$, Cohen's $d=.21$, did not significantly differ between the HL (520 ms; 21.4\%) and LH conditions (518 ms; 20.3\%; see Figure 2). Bayesian statistics were run to further test the null effects. Bayesian $t$ tests revealed anecdotal evidence for the absence of the calorie SNARC effect for both $\mathrm{RT}\left(\mathrm{BF}_{10}=0.27\right)$ and error rate $\left(\mathrm{BF}_{10}=0.35\right)$, suggesting that the likelihood of not forming an association between low (high) calories and the left (right) side is higher than forming such an association.

Experiment 1 investigated whether there is a space-calorie association that facilitates task performance when low and high calorie items are presented on the left and right sides, respectively. Romero and Biswas (2016) proposed that presenting healthy foods on the left of unhealthy foods increases the proportion of healthy food choices. As healthy food stimuli are considered low in calories, they are associated with the left side. This is in accordance with the spatial representation of the magnitudes (Romero \& Biswas, 2016). If individuals represent low and high calories on the left and right sides, respectively, this would result in faster and more accurate target detection in congruent food pairs (LH condition). However, this was not observed, the presentation of food stimulus pairs with lower calories on the left and higher calories on the right did not facilitate task performance. In other words, target detection performance was comparable for both $\mathrm{LH}$ and $\mathrm{HL}$ pairs.

Results of Experiment 1 revealed preliminary evidence on the absence of a space-calorie association. However, other possible factors should be ruled out to provide further evidence on the absence of such an association. On the basis of this, Experiment 2 was designed to test if the space-calorie association can be obtained by biasing participants' attention to a certain region and facilitate the processing of stimuli within it. Top-down factors influence attentional orienting by biasing attention to certain stimuli or spaces (Posner, 1980). In other words, the aim of Experiment 2 was to investigate whether external factors (spatial cues) can be used to form an association between the left/right space and high/low calorie food stimuli occupying that space.

\section{EXPERIMENT 2}

Exogenous and endogenous orienting mechanisms enable the allocation of attention (Posner, 1980; Ruz \& Lupiáñez, 2002). The distinc-

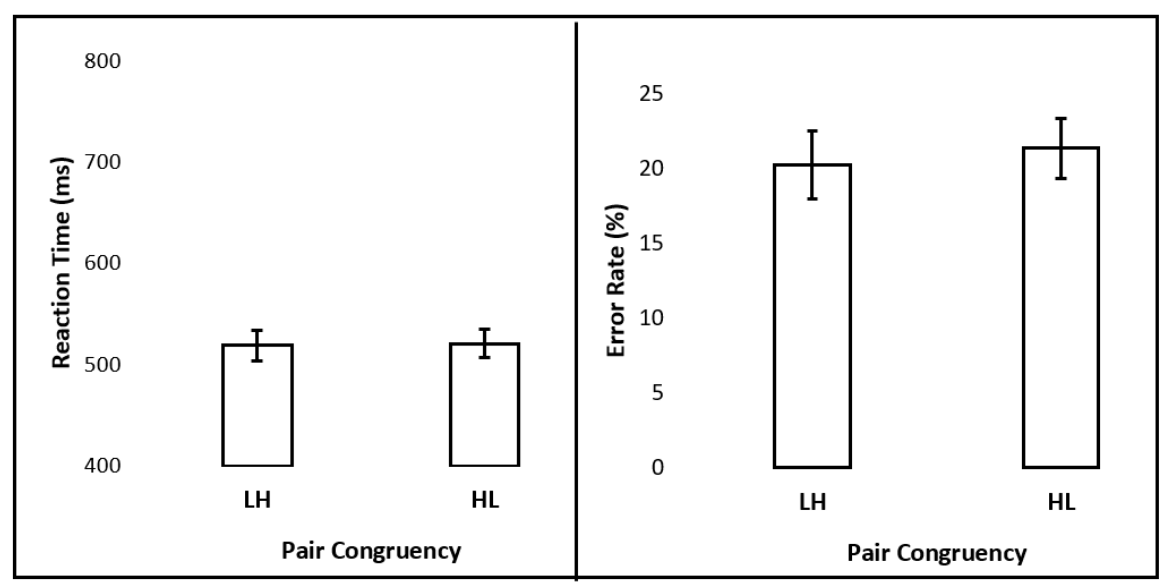

FIGURE 2.

Mean reaction time (RT, left) and error rate (right) values in the low-high $(\mathrm{LH})$ and high-low $(\mathrm{HL})$ calorie pairs in Experiment 1. The error bars represent the SEM. 
tion between the two mechanisms is based on whether the allocation of attention occurs (in)voluntarily or not. In the case of involuntary (exogenous) allocation of attention, stimulus-driven bottom-up factors are involved and, independent of the current goal, stimuli capture attention automatically by their feature. On the other hand, goal-driven top-down attentional factors affect voluntary (endogenous) allocation of attention, as attention is allocated to an object or space to fulfill a goal.

Exogenous and endogenous cues lead to different attentional processes. Exogenous (peripheral) cues are presented in the periphery and lead to reflexive and automatic orienting of attention by their sudden and brief appearance (Theeuwes, 1991). On the other hand, endogenous (central) cues are presented in the center of the screen and involve controlled orienting. Posner's spatial cueing paradigm is one of the most commonly used methods to investigate the allocation of attention in space (Posner, 1980; Posner et al., 1980). Posner (1980) presented an (in)valid spatial cue before the target onset. Presenting the target at the cued location (valid cue) or at the opposite side of the cued location (invalid cue) leads to differences in performance. Findings indicate that invalid cues lead to slower and more erroneous responses than valid cues, an effect known as reaction time cost (Posner, 1980). This is due to delayed disengagement from the invalidly cued location to the target location. On the other hand, valid cues facilitate task performance in terms of faster responses and higher accuracies, an effect known as reaction time benefit.

In Experiment 2, the aim was to investigate whether the spacecalorie association can be established in the presence of endogenous spatial cues. Spatial attention can be modulated by both numerical and non-numerical information as well as by working memory representations (van Dijck et al., 2013). Fischer et al. (2003) investigated the relationship between numbers and space. The task was based on responding to the presence of a lateralized target shape stimulus. The target was preceded by a task-irrelevant number of a small/large magnitude. Left (right) side targets were detected faster when preceded by small (large) numbers. As the presented number's magnitude modulated the spatial attention, the authors named this the attentional-SNARC effect. However, a recent replication attempt of Experiment 2 of Fischer et. al (2003) failed (Colling et al, 2019). This failed replication is an important factor to consider in the context of space-number representations. Given that the numerical stimuli do not always have spatial representations, using non-numerical stimuli such as spatial cues would be effective in investigating space-based representations. The typical spatial cueing effects reveal performance advantage of valid cues over invalid cues (Posner, 1980). If the spatial cues lead to an association between low (high) calorie and the left (right) space, the valid and invalid spatial cues would have different effects on task performance in the LH and HL conditions where locations of low/high calorie stimuli differ. In the LH condition, where low/high calorie stimuli are congruently located with regard to the space-calorie association, both valid and invalid cues would bias attention to the congruently presented stimuli (i.e., low calorie on the left, high calorie on the right side). If the cue leads to an association between calorie and space, the valid cues would facilitate performance because participants would expect to see low calorie stimulus on the left and high calorie stimulus on the right, and the valid cue would direct attention to the space-congruent item. However, the invalid cues would impair performance as attention would be directed to the non-target item. In the HL condition, where low/high calorie stimuli are incongruently located with regard to the space-calorie association, both valid and invalid cues would bias attention to stimuli that are incongruently presented in space based on the space-calorie association (e.g., high calorie on the left, low calorie on the right side) (see Figure 3). If the cue leads to an association between calorie and space, both valid and invalid cues would impair performance because participants would expect to see low calorie stimulus on the left and high calorie stimulus on the right, and both valid and invalid cues would direct attention to the space-incongruent stimuli regardless of what the target is. In the HL condition, performance should be worse in the invalid than the valid cue condition, as indicated by the typical cueing findings (Posner, 1980).

\begin{tabular}{|c|c|c|c|}
\hline Pair Congruency & Valid Cue & Invalid Cue & Space-Calorie Congruency \\
\hline \multirow{2}{*}{ LH } & & & \\
& & &
\end{tabular}

\footnotetext{
FIGURE 3.

Depiction of the spatial cue validity (valid and invalid) and pair congruency (low-high, LH, and high-low, HL) variables and (in)congruent spacecalorie associations. If the low calorie stimulus (broccoli) is the target in all cases, the valid cues (here: black arrow) would be pointing broccoli and the invalid cues would be pointing the cheesecake. Presenting the low calorie stimulus (broccoli) on the left and the high calorie stimulus (cheesecake) on the right leads to the LH pair condition and the reversed order leads to the HL pair condition. Based on the proposed space- calorie association, pair congruency determines the (in)congruency between space and calorie (LH: congruent, HL: incongruent association).
} 
If an association between space and calorie exists, valid cue trials in the LH condition would elicit better performance compared to the HL condition. When both the space-calorie association and the spatial cue are congruent (i.e., LH and valid cue), responses should be faster compared to the condition where only the cue validity is congruent but the space-calorie association is incongruent (i.e., HL and valid cue). Similar pattern would be observed in the invalid cue trials as well. When both the space-calorie association and the spatial cue are incongruent (i.e., HL and invalid cue), responses should be slower compared to when only the spatial cue is incongruent but the space-calorie association is congruent (i.e., LH and invalid cue). In other words, task performance should be worse in both valid and invalid cue trials given the incongruent space-calorie association in the HL condition.

\section{Apparatus, Stimuli, and Procedure}

In Experiment 2, the apparatus and stimuli were identical as in Experiment 1, with the exception of the presentation of the spatial cues $\left(\leftarrow\right.$ or $\rightarrow$ ) (white, size: $3.75^{\circ} \times 3^{\circ}$ ) presented prior to the food stimuli. Valid cue pointed the location of the upcoming target stimulus (e.g., right pointing cue and right-side target) and invalid cue pointed the opposite location of the target stimulus (e.g., left pointing cue and right-side target).

Each trial started with the presentation of the green or red fixation cross for $500 \mathrm{~ms}$. After the fixation cross, the (in)valid spatial cue $(\leftarrow$ or $\rightarrow$ ) was presented for $500 \mathrm{~ms}$. The fixation cross color and cue validity were randomized across trials. Following the spatial cue, food pairs were presented for $1500 \mathrm{~ms}$ and the response duration was $2000 \mathrm{~ms}$ (see Figure 4). The experiment consisted of four blocks with 120 trials each. Each block had 96 go trials (LH and HL conditions, 50-50\%) and 24 no-go trials (LL and HH conditions, 50-50\%). In the go trials, cue validity was $75 \%$ valid- $25 \%$ invalid.

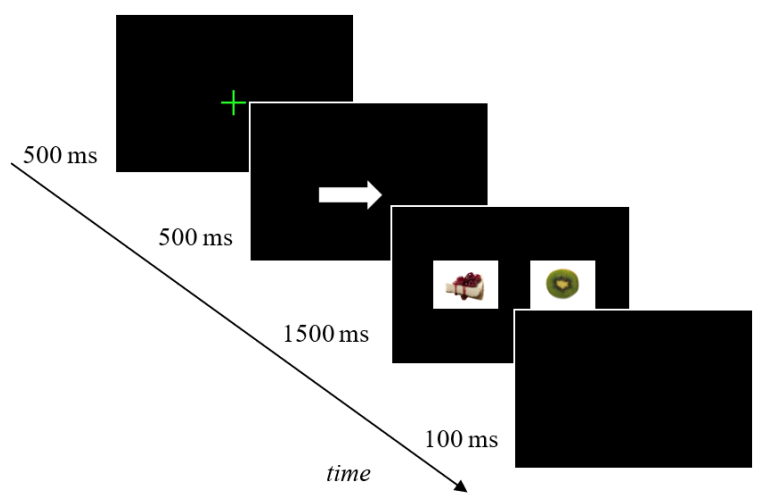

\section{FIGURE 4.}

The trial sequence of Experiment 2. Each trial started with the presentation of green or red fixation cross for $500 \mathrm{~ms}$, followed by an (in)valid spatial cue presented for 500 ms. Food stimulus pairs were presented and remained visible for $1500 \mathrm{~ms}$. The task was to indicate the location of the low (high) calorie food stimulus after the green (red) fixation cross. Here: the incongruent high-low calorie $(\mathrm{HL})$ pair was preceded by the green fixation cross and the valid spatial cue; the target was the lowcalorie food stimulus (kiwi).

\section{Results and Discussion}

Data of two participants were excluded from the analyses due to failure to follow instructions. Incorrect responses (13.17\%) and RTs outside of the range of $\pm 2.5 S D$ s from the mean were discarded as outliers (2.51\%). The no-go trials (HH and LL pairs) were not included in the analyses. 2 (pair congruency: LH, HL) $\times 2$ (cue validity: valid, invalid) repeated-measures analyses of variance (ANOVAs) were run separately for the RT and error rate measurements. Post-hoc comparisons (Tukey) were conducted for further comparisons.

Cue validity had a significant main effect on reaction time, $F(1$, 15) $=5.82, p=0.02, \eta_{\mathrm{p}}{ }^{2}=0.28$. The Bayes factor revealed anecdotal evidence in favor of the alternative hypothesis $\left(\mathrm{BF}_{10}=1.16\right)$, suggesting that the RT difference between the valid cue $(703 \mathrm{~ms})$ and invalid cue trials $(717 \mathrm{~ms})$ may not be reliable. There was no significant main effect of pair congruency, $F(1,15)=1.48, p=0.24, \eta_{\mathrm{p}}{ }^{2}=0.09$ (see Figure 5). Bayesian ANOVA results revealed evidence for the null effect of the pair congruency variable $\left(\mathrm{BF}_{10}=0.50\right)$.

Cue validity had a significant main effect on the error rate, $F(1,15)$ $=27.37, p<.001 \eta_{\mathrm{p}}{ }^{2}=.64$, supported by the Bayes factor as well $(\mathrm{BF} 10$ $=134.5)$. Invalid cues led to higher error rates (17.4\%) compared to the valid cues $(11.8 \%)$. The main effect of pair congruency on error rate was also significant, $F(1,15)=13.24, p=.002, \eta_{\mathrm{p}}{ }^{2}=.46$. Participants had higher error rates in the $\mathrm{LH}$ pair condition (16.8\%) than the HL condition (12.4\%). The Bayes factor revealed moderate evidence $\left(\mathrm{BF}_{10}\right.$ $=8.93$ ) for the effect of pair congruency. The pair congruency and cue validity interaction was significant, $F(1,15)=12.17, p=.003, \eta_{\mathrm{p}}{ }^{2}=$ .44. Participants made more errors in the LH pair condition only when the cue was invalid, contrary to our expectation. The Bayes factor revealed extreme evidence for this interaction effect $\left(\mathrm{BF}_{10}=77885.62\right)$ (see Figure 5).

Experiment 2 investigated whether the space-calorie association can be observed in the presence of endogenous spatial cues that bias attention to certain space. The obtained cue validity effect (i.e., faster and more accurate responses for the valid cues) is in accordance with previous findings (Berger et al., 1999; Blair et al., 2017; Brignani et al., 2009; Posner, 1980). If the food pairs had readily available representation (Romero \& Biswas, 2016) with low calories on the left and high calories on the right, task performance in the LH and HL conditions would have differed based on the (in)congruency between the caloriespace association. However, this was not observed; error rates were higher in the invalid cue trials in the LH but not in the HL condition, which was unexpected. This finding suggests that endogenous spatial cues do not necessarily lead to an association between space and calorie magnitude.

In Experiments 1 and 2, the color of the fixation cross varied between red and green to define the target stimulus. A red (green) fixation cross indicated that the target would be the high (low) calorie food stimulus in a given trial. This target-defining feature could have activated approach and avoidance mechanisms towards the food stimuli, resulting in slower RTs for the high-calorie food in the presence of the red fixation cross (Bruno et al., 2013). In other words, the red fixation cross might have created a stop signal, whereas the green 


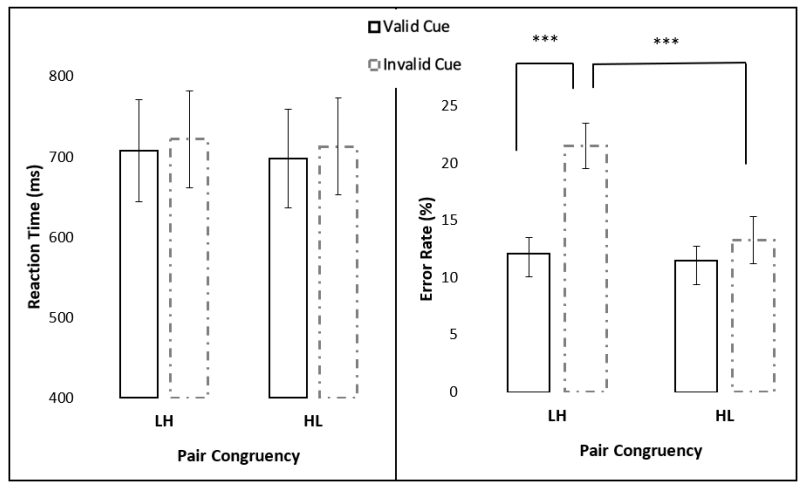

FIGURE 5.

Mean reaction time (RT, left) and error rate (right) values in the low-high $(\mathrm{LH})$ and high-low $(\mathrm{HL})$ calorie pairs in Experiment 2. The error bars represent the SEM $\left({ }^{* * *} p<.001\right)$

fixation cross might have created a go signal towards the food stimuli. While approach behaviors are demonstrated by moving towards the positive stimulus, avoidance behaviors are considered as moving away from the negative stimulus (Elliot et al., 2009). Approach-avoidance behaviors towards food stimuli under different color conditions were investigated in the food context as well. Bruno et al. (2013) investigated the effects of colors on food choice behavior. They served popcorn (Experiment 1) and chocolate chips (Experiment 2) to different group of participants on red, blue, and white plates. Participants were asked to try the food items and then evaluate their sensory qualities with a mock questionnaire. Participants consumed fewer popcorns and chocolate chips when they were served on a red plate compared to the blue or white plates. The authors interpreted this as red denoting danger and prohibition, which is a culturally learned or biologically embedded association (see also Genschow et al., 2012, for similar results). To sum up, the current literature indicates that the color red evokes avoidance behavior, which leads to decreased food intake. Experiment 3 was designed to investigate if the color red evokes avoidance motivation towards the food stimuli and affects the association between space and calorie.

\section{EXPERIMENT 3}

Rohr et al. (2015) examined the approach-avoidance behaviors in the food domain with green and red colors. Participants were presented with black and white line drawings of healthy and unhealthy foods surrounded by task-irrelevant red or green circles. They were instructed to move the mouse towards themselves (approach behavior) whenever they saw heathy food drawing or away from themselves (avoidance behavior) whenever they saw unhealthy food drawing, regardless of the surrounding circle's color. Stimulus-response mappings were reversed in the half of the blocks. When participants were asked to move the mouse away in the presence of the unhealthy food stimuli, responses were faster on the trials with red circles compared to green circles. Therefore, the authors concluded that the color red increased avoidance behavior towards unhealthy food stimuli while the color green did not lead to approach behavior towards the healthy food items (Rohr et al., 2015). Applied to the present study, activation of an automatic pattern might have resulted in avoidance behavior for the high-calorie target stimuli followed by the red fixation cross. Avoiding the high calorie target could have prevented the formation of space-calorie association, and this needs to be controlled for. In order to examine whether the red color activates such a pattern, Experiment 3 was conducted to examine whether the fixation cross color activates approach and avoidance mechanisms for the low and high calorie stimuli, respectively. In Experiment 3, the fixation cross color was task-irrelevant and was randomized across trials. In other words, the color of the fixation cross did not define the target stimuli as in the previous experiments. The target was determined block-wise and participants were informed about the target item (low/high calorie) prior to each block. Therefore, in the current experiment, if the color red (green) evokes avoidance (approach) behavior, participants would respond slower (faster) to the high (low) calorie food stimuli.

\section{Apparatus, Stimuli, and Procedure}

Materials used in Experiment 3 were similar to Experiment 1, with the exception of the selection criteria of the food stimuli. In Experiment 1, the visual properties such as complexity and object size of the stimuli were not controlled which may have been confounding. In Experiment 3 , in order to create low and high calorie food stimulus sets, a subsample of images presented in Experiment 1 with few additional new ones were selected and the object size and complexity scores of the food stimuli were matched. Scores for object size and complexity were obtained from normative ratings of the Food-pics database (Blechert et al., 2019; Blechert et al., 2019). Stimuli with the scores $\pm 1 S D$ from the mean score of complexity and object size were discarded from the stimuli set, leading to 16 low and high calorie stimuli each.

The procedure of Experiment 3 was identical to Experiment 1, except that the fixation cross color was task-irrelevant and did not define the target stimulus. The target was the low/high calorie food stimulus in $50-50 \%$ probability across blocks. Each target was presented blockwise and the order of the blocks was counterbalanced. The approach and avoidance behaviors were measured by the executed responses. In 
Experiment 3, the no-go trials were not included, as they do not require any response. The practice block was identical with Experiment, 1 except that the participants were instructed to indicate pebbles' location since the target stimuli did not change within the block as in Experiment 1. Each trial started with the presentation of red or green fixation cross for $500 \mathrm{~ms}$. The food pairs ( $\mathrm{LH}$ or $\mathrm{HL}$ ) were presented for $1500 \mathrm{~ms}$. Participants were asked to press the "A" or " $\mathrm{L}$ " buttons for the left/right side targets, respectively. The response duration was $2000 \mathrm{~ms}$. The background color was white. The experiment consisted of 4 blocks with 120 trials each.

\section{Results and Discussion}

Data of one participant were excluded from the analyses due to failure to follow instructions. Incorrect responses (5.14\%) and RTs outside of the range of $\pm 2.5 S D$ s from the mean were discarded as outliers (2.68\%). 2 (fixation cross color: red, green) $\times 2$ (pair congruency: $\mathrm{LH}$, $\mathrm{HL}) \times 2$ (target's calorie level: low, high) repeated-measures ANOVA was conducted separately for RT and error rate measurements. The detection of low/high calorie targets followed by the red or green fixation cross was of particular interest to investigate the approach/avoidance mechanisms.

The RT analyses did not reveal any effects of the fixation cross color, $F(1,17)=2.16, p=.15, \eta_{\mathrm{p}}^{2}=0.11$, pair congruency, $F(1,17)=2.76$, $p=.11 \eta_{\mathrm{p}}{ }^{2}=0.14$, nor target's calorie level, $F(1,17)=0.15, p=.69, \eta_{\mathrm{p}}{ }^{2}$ $=0.009$. Bayesian ANOVA results revealed anecdotal evidence for the effects of fixation cross color $\left(\mathrm{BF}_{10}=0.27\right)$, pair congruency $\left(\mathrm{BF}_{10}=\right.$ $0.21)$, and target calorie $\left(\mathrm{BF}_{10}=0.23\right)$. Similar pattern was observed for the error rates. No significant effect of pair congruency, $F(1,17)=$ $0.28, p=.60, \eta_{\mathrm{p}}{ }^{2}=0.01$, fixation cross color, $F(1,17)=0.38, p=0.54$, $\eta_{\mathrm{p}}{ }^{2}=0.02$, and target calorie level, $F(1,17)=0.43, p=0.51, \eta_{\mathrm{p}}{ }^{2}=0.02$, was observed on error rates (see Figure 6). The Bayes factor revealed anecdotal evidence for the effects of fixation cross color $\left(\mathrm{BF}_{10}=0.19\right)$, pair congruency $\left(\mathrm{BF}_{10}=0.22\right)$, and target calorie $\left(\mathrm{BF}_{10}=0.26\right)$. Results suggest that target detection performance is not modulated by any of the factors.

Experiment 3 was designed to test whether the lack of the spacecalorie association is due to the presentation of red and green fixation crosses activating the avoidance and approach mechanisms, respectively. The activation of such mechanisms could potentially prevent the formation of the space-calorie association. However, results did not reveal any differences in the detection of low versus high calorie targets followed by the red or green fixation crosses. The use of task-irrelevant red fixation cross did not result in avoiding the high calorie stimuli (i.e., slowing the target selection process), which contradicts the findings of Rohr et al. (2015). Overall, the findings of Experiment 3 indicate that the approach and avoidance mechanisms do not account for the absence of the space-calorie association.

\section{GENERAL DISCUSSION}

The aim of the present study was to examine the space-calorie association, that is, whether lower/higher calorie stimuli are processed faster and more accurately when presented in left/right hemispace, respectively. Such presentation of food stimuli would be compatible with the space-magnitude association, albeit using the calorie magnitude. The presence of an association between space and calorie is expected to lead to representation of lower (higher) calorie magnitudes on the left (right) side. In three experiments, the existence of space-calorie association was investigated with various variables. Findings of Experiment 1 revealed that participants do not necessarily form an association between calorie magnitudes and as they would with the mental number line. Experiment 2 was designed to further investigate whether topdown spatial attention mechanisms would contribute to the formation of the space-calorie association. Presenting (in)valid endogenous spatial cues did not lead to an space-calorie association. In Experiments 1 and 2, the color of the fixation cross determined the target to be detected. The nature of the task could have led to an association between the color red and the high calorie stimulus. Previously, an association between avoidance response towards unhealthy food and the color red was reported (Rohr et al., 2015). This may have accounted for the findings obtained in Experiments 1 and 2. The color red could have activated avoidance behavior and affected the performance on trials where high calorie stimulus was the target, which prevented the formation of the space-calorie association. Experiment 3 was conducted to control the possible role of the avoidance mechanism. The color of the fixation cross did not determine the target and both low and high calorie stimuli were preceded by either red or green fixation cross in

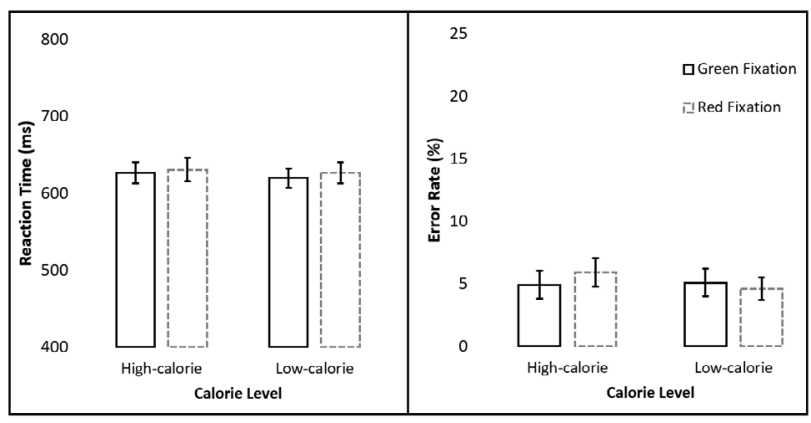

FIGURE 6.

Mean reaction time (RT, left) and error rate (right) values for high and low calorie targets presented after red or green fixation cross conditions in Experiment 3. The error bars represent the SEM. 
equal proportion. Implementing such a design was expected to prevent the activation of the avoidance mechanism. Findings again did not reveal any evidence for the space-calorie association. Overall, the current (preliminary) findings indicate that the non-numerical SNARC effect cannot be generalized to the calorie domain, unlike the musical (Cho et al., 2012), temporal (Gevers et al., 2003), size (Dural et al., 2018), or luminance (Ren et al., 2011) domains.

The current results indicate the lack of an association between space and the calorie level. However, certain limitations should be addressed which could account for the results. Information on participants' current satiety level, weight, body mass index, eating disorders, or dietary restrictions were not collected. These factors could have affected the processing of the food stimuli. Previously, it has been reported that food deprivation prolongs attentional dwell time for food stimuli (Mogg et al., 1998; Placanica et al., 2002) or modulates the approach behavior towards food stimuli (Seibt et al., 2007). These factors should be taken into account in the future studies to rule out potential confounding factors.

The current findings may as well depend on the task properties. Previously, a space-calorie association was formed (i.e., representing higher calories on the right side) when a single stimulus was presented and the task was based on categorizing the food stimulus as low/ high calorie (Manippa et al., 2020, Experiment 1). However, when two stimuli were presented simultaneously (Romero \& Biswas 2016; Manippa et al., 2020, Experiment 4) and participants were asked to choose one out of the two options, the calorie-space association was replaced by the healthiness and space association as participants prioritized healthiness over the calorie domain (i.e., formation of healthy food-left side association). This could indicate the role of task demands (i.e., processing single vs. multiple stimuli) and task-relevant dimensions (judgment of the calorie level or choosing the healthier item) on the (lack of) formation of an association. In the present study, participants were presented with food stimuli pairs and were asked to make a forced choice among the two alternatives. However, we did not control whether participants performed the task by focusing on the healthiness aspect rather than the calorie level. Based on the results of Manippa et al. (2020), it may be possible that our participants prioritized the healthiness aspect of the presented stimuli over the calorie level. Such a pattern could explain the lack of the space-calorie association. Given the ambiguities on which aspect (healthiness or calorie level) was exactly involved and the role of presentation format (single vs. multiple stimuli) on the results, different tasks could be implemented in the future studies. On the other hand, one could also argue whether reaction time tasks are suitable to investigate the space-based representations (see Manippa et al., 2020, Experiment 3, where pleasantness of the food stimuli was investigated in a reaction time task and no association was formed between space and pleasantness).

Another critical factor is the difference in the ratings of the selected food stimuli on the arousal and valence parameters. In Experiments 1 and 2, low calorie stimuli (59) had higher valence ratings than the high calorie stimuli (53.7), and high calorie stimuli (37) were more arousing than the low calorie stimuli (34.2, see also Table 1$)$. The researchers who created the Food-pics database operationalized valence as the food item being repulsive (negative valence) or attractive (positive valence) for the participant. Arousal was operationalized as the level of the elicited emotional reaction by the presented stimulus (Blechert et al., 2019; Blechert et al., 2014). In Experiments 1 and 2, the selected low calorie stimuli were more attractive than the high calorie stimuli and somehow high calorie stimuli elicited stronger emotional reaction than the low calorie stimuli. Given the nonsystematic (or reversed) variance in the ratings of the low and high calorie stimuli, it is not possible to link the pattern in the current results to the differences in valence and arousal ratings. The selected culturally-familiar pictures (see the pilot study) varied in the aforementioned parameters. However, it should be noted that in Experiment 3, the selected images were matched on the arousal and valence parameters and the results again revealed the lack of an association between space and the calorie magnitude. Furthermore, and most importantly, palatability and craving ratings of the low and high calorie stimuli were matched in all experiments.

The final remark is on the participants' implicit, culture-specific associations. In some cultures, like where this study was conducted, good and bad concepts are associated with right and left sides, respectively. Such implicit associations may prevent to form an association between low (good) calorie and high (bad) calorie items and left-right sides. In other words, implicit associations may prevent to associate low calorie items with the left side because although low calorie is the healthy option, it may go against the survival needs where low represents less. Supporting evidence on this claim comes from the body-specificity hypothesis, according to which individuals' handedness and mental representation of positive and negative concepts are related. People associate good or positive concepts with the hemispace that their hand is dominant in (Casasanto, 2009). That is, right-handed people associate positive valence with the right hemispace and negative valence with the left hemispace. The reversed pattern is observed for left-handed people (i.e., positive valence is associated with the left hemispace). Given that the vast majority of this study's sample was right-handed, such an implicit mechanism could also have a role in the failed representations between space and the calorie magnitude.

In conclusion, based on the reaction time performance, our results revealed preliminary evidence on the lack of an association between low (high) calorie and left (right) space, unlike other SNARC-like effects observed in various domains. Future studies could further explore the representation of the calorie magnitude in space.

\section{REFERENCES}

Berger, A., Dori, H., \& Henik, A. (1999). Peripheral non-informative cues do induce early facilitation of target detection. European Journal of Cognitive Psychology, 11, 119-137. doi: 10.1080/713752304 الم

Blair, C., Capozzi, F., \& Ristic, J. (2017). Where is your attention? Assessing individual instances of covert attentional orienting in response to gaze and arrow cues. Vision, 1, 1-13. doi: 10.3390/vision1030019

Blechert, J., Lender, A., Polk, S., Busch, N., \& Ohla, K. (2019). Foodpics_extended-an image database for experimental research on 
eating and appetite: additional images, normative ratings and an updated review. Frontiers in Psychology, 10, 307. doi: 10.3389/fpsyg.2019.00307 الفلسلس

Blechert, J., Meule, A., Busch, N. A., \& Ohla, K. (2014). Food-pics: An image database for experimental research on eating and appetite. Frontiers in Psychology, 5, 617. doi: 10.3389/fpsyg.2014.00617 المسلسلس

Brignani, D., Guzzon, D., Marzi, C. A., \& Miniussi, C. (2009). Attentional orienting induced by arrows and eye-gaze compared with an endogenous cue. Neuropsychologia, 47, 370-381. doi: 10.1016/j.neuropsychologia.2008.09.011 السلبلسلة

Bruno, N., Martani, M., Corsini, C., \& Oleari, C. (2013). The effect of the color red on consuming food does not depend on achromatic (Michelson) contrast and extends to rubbing cream on the skin. Appetite, 71, 307-313. doi: 10.1016/j.appet.2013.08.012 البلسلسلسا

Casasanto, D. (2009). Embodiment of abstract concepts: good and bad in right-and left- handers. Journal of Experimental Psychology: General, 138, 351-367. doi: 10.1037/a0015854 سلسلس

Chandon, P., \& Wansink, B. (2007). The biasing health halos of fastfood restaurant health claims: Lower calorie estimates and higher side-dish consumption intentions. Journal of Consumer Research,

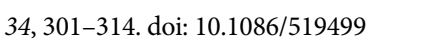

Cho, Y. S., Bae, G. Y., \& Proctor, R. W. (2012). Referential coding contributes to the horizontal SMARC effect. Journal of Experimental Psychology: Human Perception and Performance, 38, 726-734. doi: 10.1037/a0026157 سلس

Colling, L. J., Szűcs, D., De Marco, D., Cipora, K., Ulrich, R., Nuerk, H. C., ... \& Henare, D. T. (2019). Registered replication report on Fischer, Castel, Dodd, And Pratt (2003). Advances in Methods and Practices in Psychological Science, 2515245920903079. المالمسلس

Dehaene, S., Bossini, S., \& Giraux, P. (1993). The mental representation of parity and number magnitude. Journal of Experimental Psychology: General, 122, 371-396. doi: 10.1037/0096-3445.122.3.371 المالسلسلة

Deng, X., \& Kahn, B. E. (2009). Is your product on the right side? The "location effect" on perceived product heaviness and package evaluation. Journal of Marketing Research, 46, 725-738. doi: 10.1509/ jmkr.46.6.725_JMR6B سلس للس

Dural, S., Burhanoğlu, B. B., Ekinci, N., Gürbüz, E., Akın, İ. U., Can, S., \& Çetinkaya, H. (2018). Compatibility between physical stimulus size-spatial position and false recognitions. Frontiers in Psychology, 9, 1457. doi: 10.3389/fpsyg.2018.01457 المالسلس

Elliot, A. J., Maier, M. A., Binser, M. J., Friedman, R., \& Pekrun, R. (2009). The effect of red on avoidance behavior in achievement contexts. Personality and Social Psychology Bulletin, 35, 365-375. doi: 10.1177/0146167208328330 الس الس

Fischer, M. H., Castel, A. D., Dodd, M. D., \& Pratt, J. (2003). Perceiving numbers causes spatial shifts of attention. Nature Neuroscience, 6,

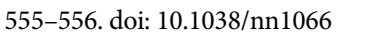

Fleiss, J. L. (1971). Measuring nominal scale agreement among many raters. Psychological Bulletin, 76, 378-382. doi: 10.1037/h0031619 الملسلسلس

Fumarola, A., Prpic, V., Da Pos, O., Murgia, M., Umiltà, C., \& Agostini, T. (2014). Automatic spatial association for luminance. Attention, Perception, \& Psychophysics, 76, 759-765. doi: 10.3758/s13414-013- 0614-y الم

Genschow, O., Reutner, L., \& Wänke, M. (2012). The color red reduces snack food and soft drink intake. Appetite, 58, 699-702. doi:

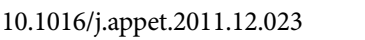

Gevers, W., Reynvoet, B., \& Fias, W. (2003). The mental representation of ordinal sequences is spatially organized. Cognition, 87, B87-B95.

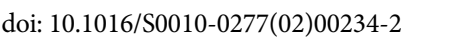

JASP Team (2019). JASP (Version 0.11.1) [Computer software] السلسلس Landis, J. R., \& Koch, G. G. (1977). The measurement of observer agreement for categorical data. Biometrics, 159-174. doi:

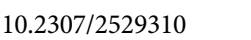

Macnamara, A., Keage, H. A., \& Loetscher, T. (2018). Mapping of non-numerical domains on space: A systematic review and metaanalysis. Experimental Brain Research, 236, 335-346. doi: 10.1007/

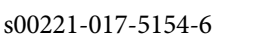

Manippa, V., Giuliani, F., \& Brancucci, A. (2020). Healthiness or calories? Side biases in food perception and preference. Appetite, 147,

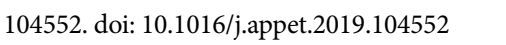

Marsman, M., \& Wagenmakers, E. J. (2017). Bayesian benefits with JASP. European Journal of Developmental Psychology, 14, 545-555.

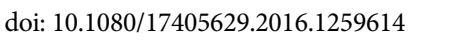

Meyer, D. E., Osman, A. M., Irwin, D. E., \& Yantis, S. (1988). Modern mental chronometry. Biological Psychology, 26, 3-67. doi: 10.1016/0301-0511(88)90013-0 الس الس الس

Mogg, K., Bradley, B. P., Hyare, H., \& Lee, S. (1998). Selective attention to food-related stimuli in hunger: Are attentional biases specific to emotional and psychopathological states, or are they also found in normal drive states? Behaviour Research and Therapy, 36, 227-237. doi: 10.1016/S0005-7967(97)00062-4 السليلسلة

Moyer, R. S., \& Landauer, T. K. (1967). Time required for judgements of numerical inequality. Nature, 215(5109), 1519-1520. doi: 10.1038/2151519a0 布

Nishimura, A., \& Yokosawa, K. (2009). Effects of laterality and pitch height of an auditory accessory stimulus on horizontal response selection: The Simon effect and the SMARC effect. Psychonomic Bulletin \& Review, 16, 666-670. doi: 10.3758/PBR.16.4.666 له

Peirce, J. W. (2007). PsychoPy-psychophysics software in Python. Journal of Neuroscience Methods, 162, 8-13. doi: 10.1016/j.jneu-

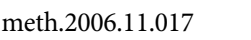

Peirce, J. W. (2009). Generating stimuli for neuroscience using PsychoPy. Frontiers in Neuroinformatics, 2, 10. doi: 10.3389/neu-

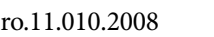

Placanica, J. L., Faunce, G. J., \& Soames Job, R. F. (2002). The effect of fasting on attentional biases for food and body shape/weight words in high and low eating disorder inventory scorers. International Journal of Eating Disorders, 32, 79-90. doi: 10.1002/eat.10066 الفلسلس

Posner, M. I. (1980). Orienting of attention. Quarterly Journal of Experimental Psychology, 32, 3-25. doi: 10.1080/00335558008248231 إلسلس

Posner, M. I., Snyder, C. R., \& Davidson, B. J. (1980). Attention and the detection of signals. Journal of Experimental Psychology: General,

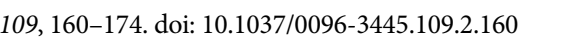

Proctor, R. W., \& Cho, Y. S. (2006). Polarity correspondence: A gen- 
eral principle for performance of speeded binary classification tasks. Psychological Bulletin, 132, 416-442. doi: 10.1037/0033-

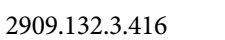

R Development Core Team. (2017). R: A language and environment for statistical computing [Computer software]. R Foundation for Statistical Computing, Vienna, Austria. الس الس

Ren, P., Nicholls, M. E., Ma, Y. Y., \& Chen, L. (2011). Size matters: Non-numerical magnitude affects the spatial coding of response. PLoS One, 6, e23553. doi: https://journals.plos.org/plosone/ article?id=10.1371/journal.pone.0023553 المالسلسل

Rohr, M., Kamm, F., Koenigstorfer, J., Groeppel-Klein, A., \& Wentura, D. (2015). The color red supports avoidance reactions to unhealthy food. Experimental Psychology, 62, 335-345. doi: 10.1371/journal. pone.0023553 الس

Romero, M., \& Biswas, D. (2016). Healthy-left, unhealthy-right: Can displaying healthy items to the left (versus right) of unhealthy items nudge healthier choices? Journal of Consumer Research, 43, 103-112. doi: 10.1093/jcr/ucw008 每

Rozin, P., Scott, S., Dingley, M., Urbanek, J. K., Jiang, H., \& Kaltenbach, M. (2011). Nudge to nobesity I: Minor changes in accessibility decrease food intake. Judgment and Decision Making, 6, 323-332.

Ruz, M., \& Lupiáñez, J. (2002). A review of attentional capture: On its automaticity and sensitivity to endogenous control. Psicológica, 23, 283-309. |لسلسل

Seibt, B., Häfner, M., \& Deutsch, R. (2007). Prepared to eat: How immediate affective and motivational responses to food cues are influenced by food deprivation. European Journal of Social Psychology, 37, 359-379. doi: 10.1002/ejsp.365 السلسلس

Sellaro, R., Treccani, B., Job, R., \& Cubelli, R. (2015). Spatial coding of object typical size: evidence for a SNARC-like effect. Psychological Research, 79, 950-962. doi: 10.1007/s00426-014-0636-7 السلسلس
The jamovi project (2019). jamovi (Version 0.9) [Computer software]. |سلس

Theeuwes, J. (1991). Exogenous and endogenous control of attention: The effect of visual onsets and offsets. Perception \& Psychophysics, 49, 83-90. doi: 10.3758/BF03211619 المسلسلس

Torralbo, A., Santiago, J., \& Lupiáñez, J. (2006). Flexible conceptual projection of time onto spatial frames of reference. Cognitive Science, 30, 745-757. doi: 10.1207/s15516709cog0000_67 المبلسلس

Tversky, B. (2011). Spatial thought, social thought. In T. W. Schubert \& A. Maass (Eds.), Spatial dimensions of social thought (Vol. 18, pp. 17-38). de Gruyter.

Valenzuela, A., \& Raghubir, P. (2015). Are consumers aware of topbottom but not of left-right inferences? Implications for shelf space positions. Journal of Experimental Psychology: Applied, 21, 224-241.

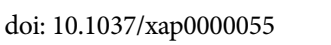

van Dijck, J. P., \& Fias, W. (2011). A working memory account for spatial-numerical associations. Cognition, 119, 114-119. doi: 10.1016/j. cognition.2010.12.013 المالسلس

van Dijck, J. P., Abrahamse, E. L., Majerus, S., \& Fias, W. (2013). Spatial attention interacts with serial-order retrieval from verbal working memory. Psychological Science, 24, 1854-1859. doi: 10.1177/0956797613479610 البلإلسلس

Wood, G., Willmes, K., Nuerk, H.-C., \& Fischer, M. H. (2008). On the cognitive link between space and number: A meta-analysis of the SNARC effect. Psychology Science, 50, 489-525. السلسلسل| 


\section{APPENDIX A}

\section{IDs of the Stimuli Used in the Present Study Taken from the Food-pics Database}

\section{Experiments 1 and 2 low-calorie stimuli:}

186, 194, 196, 197, 198, 199, 200, 201, 202, 203, 204, 206, 208, 210, 215 , $216,221,222,224,241,243,246,247,249,250,251,252,253,255,256$, $257,258,259,260,263,264,265,266,267,269,270,275,276,280,281$, $282,283,284,285,288,305,321,333,335,342,356,380,382,386,389$, $391,396,398,401,407,413,429,438,453,454,528,558,560,566,567$, $761,797,832,833,868$.

Experiments 1 and 2 high-calorie stimuli:

$1,2,4,5,6,9,12,15,16,20,22,24,25,26,29,30,32,34,38,41,42,46$, $48,49,52,57,59,61,68,75,77,79,81,83,97,101,104,105,108,111$, $113,117,123,125,130,138,140,157,158,159,162,163,166,172,174$, $188,189,287,289,290,293,295,296,302,313,363,375,387,400,421$, $484,485,488,492,510,546,565,669,810,825$.

Experiment 3 low and high-calorie stimuli:

Low-calorie: 194, 221, 247, 249, 252, 257, 335, 360, 444, 446, 453, 458, $468,530,569,793$.

High-calorie: $9,16,18,22,30,46,61,79,87,127,138,174,290,328$, 400,510 . 